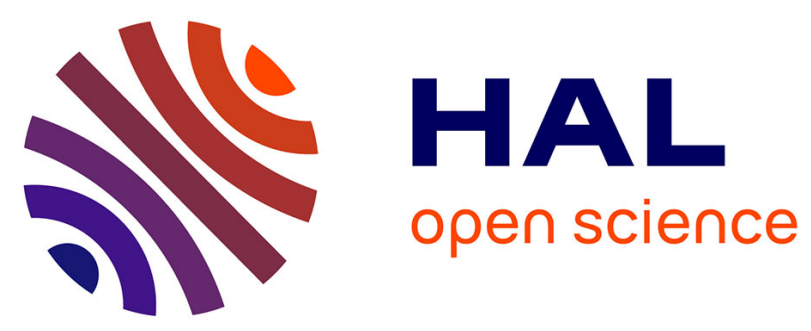

\title{
MRI-guided clinical 6-MV radiosensitization of glioma using a unique gadolinium-based nanoparticles injection
}

Camille Verry, Sandrine Dufort, Emmanuel Luc Barbier, Olivier Montigon, Michel Peoc'H, Philippe Chartier, François Lux, Jacques Balosso, Olivier Tillement, Lucie Sancey, et al.

\section{To cite this version:}

Camille Verry, Sandrine Dufort, Emmanuel Luc Barbier, Olivier Montigon, Michel Peoc'H, et al.. MRI-guided clinical 6-MV radiosensitization of glioma using a unique gadolinium-based nanoparticles injection. Nanomedicine, 2016, 11 (18), pp.2405-2417. 10.2217/nnm-2016-0203 . hal-01405486

\author{
HAL Id: hal-01405486 \\ https://hal.science/hal-01405486
}

Submitted on 5 Feb 2021

HAL is a multi-disciplinary open access archive for the deposit and dissemination of scientific research documents, whether they are published or not. The documents may come from teaching and research institutions in France or abroad, or from public or private research centers.
L'archive ouverte pluridisciplinaire HAL, est destinée au dépôt et à la diffusion de documents scientifiques de niveau recherche, publiés ou non, émanant des établissements d'enseignement et de recherche français ou étrangers, des laboratoires publics ou privés. 


\section{MRI-guided clinical 6-MV radiosensitization of glioma using a unique gadolinium- based nanoparticles injection}

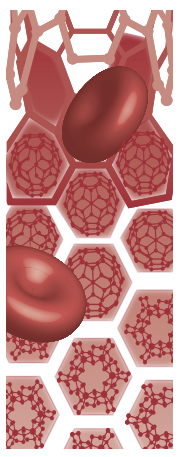

\begin{abstract}
Aim: This study reports the use of gadolinium-based AGuIX nanoparticles (NPs) as a theranostic tool for both image-guided radiation therapy and radiosensitization of brain tumors. Materials \& methods: Pharmacokinetics and regulatory toxicology investigations were performed on rodents. The AGuIX NPs' tumor accumulation was studied by MRI before 6-MV irradiation. Results: AGuIX NPs exhibited a great safety profile. A single intravenous administration enabled the tumor delineation by MRI with a $\mathrm{T}_{1}$ tumor contrast enhancement up to $24 \mathrm{~h}$, and the tumor volume reduction when combined with a clinical 6-MV radiotherapy. Conclusion: This study demonstrates the efficacy and the potential of AGuIX NPs for image-guided radiation therapy, promising properties that will be assessed in the upcoming Phase I clinical trial.
\end{abstract}

First draft submitted: 17 May 2016; Accepted for publication: 20 July 2016; Published online: 16 August 2016

Keywords: clinical 6-MV radiation therapy $\bullet$ MRI-guided radiation therapy $\bullet$ radiosensitizing nanoparticles

Glioma are the most frequent primary tumors of the CNS (70\%). Among them, glioblastoma is the most prevalent and aggressive malignant type in adults (around 60\% among glioma). Despite a standard treatment combining surgery, chemotherapy and radiotherapy, tumor recurrences are common, leading to a median survival time around 1 year after diagnosis, and a poor 5 -year survival of only $9.8 \%$ [1]. Standard therapy optimizations, as well as new therapeutics, are urgently needed to improve the outcome of patients with glioblastoma.

Although radiotherapy is presently the most efficient treatment against brain tumors, it is primarily limited in its ability to deliver therapeutic doses to target tumor volume while minimizing damages to the surrounding healthy tissues. In addition to the modulation of radiation exposure parameters and/or geometry $[2,3]$ and to the combination with chemotherapy, one attractive approach to overcome this limitation is the use of new therapeutic agents (molecules or nanoparticles [NPs]) that sensitize cancer cells to ionizing radiation (i.e., dose enhancers and radiosensitizers). In particular, the concept of the dose enhancers is to increase the radiobiological effect of high-energy radiation inside the tumor by incorporating high-density and high atomic number elements thus locally significantly increasing the dose effect delivered by the particles beams [4-8]. This enhanced local absorption in presence of high Z-elements nearby the irradiated target volume translates into a larger production of harmful diffused photons, photoelectrons, Auger electrons, Compton electrons and radical species [9]. The higher local energy absorption and the larger production of reactive species should contribute to severe tumor damages, in other words, a more efficient destruction of the tumor, without modifying the delivered dose
Camille, Verry $y^{1,2,3}$, Sandrine Dufort ${ }^{4,8}$, Emmanuel Luc Barbier $^{2,3}$, Olivier Montigon ${ }^{2,3}$, Michel Peoc' $h^{5}$, Philippe Chartier ${ }^{1}$, François Lux ${ }^{6}$, Jacques Balosso', Olivier Tillement ${ }^{6}$, Lucie Sancey ${ }^{6}$ \& Géraldine Le Duc*7,8

'Department of Radiotherapy, Grenoble Alpes University Hospital, BP217, F38043 Grenoble, Cedex 9, France ${ }^{2}$ Grenoble Institute of Neurosciences, Grenoble Alpes University, F38000 Grenoble, France IINSERM U1216, F38000 Grenoble, France

${ }^{4}$ Nano-H SAS, F38070 Saint-QuentinFallavier, France

${ }^{5}$ Department of Pathology, Saint-Etienne University Hospital, F42055 SaintEtienne, Cedex 2, France

${ }^{6}$ Institute Light \& Mater, UMR5306, Lyon1 University-CNRS, Lyon University, F69622 Villeurbanne, France

${ }^{7}$ Biomedical Beamline, European Synchrotron Radiation Facility, CS40220, F38043 Grenoble, Cedex 9, France ${ }^{8}$ Present affiliation: NH TherAguix, 43 boulevard du 11 novembre 1918, F69100 Villeurbanne, France *Author for correspondence: Tel.: +33 (6) 87121040 leduc@esrffr

${ }^{\ddagger}$ Authors contributed equally

\section{Future Medicine


to organs at risk. Although many molecules containing high Z-elements (e.g., iodinated molecules initially conceived as contrast agents for $\mathrm{x}$-ray imaging) have been developed and assessed, the results were not satisfying because the amount of radiosensitizers in terms of number of compounds or high Z-elements within the tumors was insufficient $[10,11]$. Recently, it has been shown that high Z-NPs can overcome this limitation due to nanoscale dose deposition that will contribute indirectly to an increase in the dose within the tumor tissues [12].

In this context, we developed and formulated theranostic ultrasmall $(3.0 \pm 1.0 \mathrm{~nm}$ size $)$ gadolinium (Gd)-based AGuIX (activated guided irradiation by x-ray) NPs, which can be used for tumor delineation (by MRI) as well as for therapeutic applications (as a radiosensitizer agent) [13,14]. The AGuIX NPs are characterized by a rapid clearance, a weak tumor-to-healthy tissue ratio and by an extravasation from normal blood vessels that impedes a preferential accumulation in solid tumors which is essentially based on enhanced permeability and retention effect [15-20]. Moreover once in the tumor, the AGuIX NPs were in part internalized in the tumor cells [21]. They were very well suited for enhancing the tumor accumulation compared with $\mathrm{Gd}$ chelates, as visible on MRI images [22]. Moreover, using low-energy radiation, several studies have demonstrated the strong efficacy of the combination of AGuIX NPs and radiation exposure in tumor treatment, such as for glioblastoma [23], lung tumors [24], aggressive pancreatic adenocarcinoma [25] and multiple brain melanoma metastases [21].

Altogether, the multifunctional AGuIX NPs appear very well suited for achieving image-guided therapy. The possibility of monitoring the biodistribution by MRI is indeed crucial for optimizing the effect of radiotherapy since the radiation exposure can be triggered only when the Gd content deduced from MR images is both high in the tumor and low in the surrounding healthy tissue. Moreover, the clustering of Gd atoms within the NPs allows the heterogeneous ionizing radiation after $\mathrm{x}$-ray exposure with a nanoscale intense dose deposition; phenomenon that is not observed for the Gd-molecular compounds. Finally, the significant persistence of the NPs once trapped in the tumor could allow to perform the MRI followed by the radiotherapy using the same NPs' injection [14].

This study is the first reporting that a unique injection allows in a same protocol both the kinetic follow-up of the NPs by MRI and the radiosensitization performed with a clinical (6-MV) radiation exposure. Especially, the study includes the determination of the Gd content ratio between the tumor and the surrounding tissues as a key parameter for determining the optimal delay between the MRI and the radiotherapy session. Finally, pharmacokinetics and regulatory toxicology studies demonstrate the safety profile of the NPs for their upcoming assessment in clinical trials.

\section{Materials \& methods \\ Animals}

For MRI and radiotherapy, all procedures related to animal care conformed to the Guidelines of the European and French Government with licenses 380325 and B3818510002, and were approved by the Ethical Committee of the ESRF (ETHAX) and registered under number 01261.01 at French Ministry of Research. Pharmacokinetics and regulatory toxicology study were performed by Wil Research (protocol registered as 'RatSouris_Tox subchronique_2012dec18 cea; Wil Research, St Germain Nuelles, France).

\section{Pharmacokinetics \& regulatory toxicology study}

Wistar Han IGS: Crl: WI (Han) rats (16 males and 16 females per condition) were injected intravenously (iv.) with AGuIX NPs (7 ml/ $\mathrm{kg} / \mathrm{administration})$ on days 0 and 6 , and sacrificed 1 (10 animals/group/ sex) or 10 weeks (6 animals/group/sex) after injection (Wil Research). The administered doses were 0, 250, 500 and $750 \mathrm{mg} / \mathrm{kg} / \mathrm{administration}$, in other words, at a concentration of $0,36,71$ and $107 \mathrm{~g} / \mathrm{l}$ of AGuIX, respectively. The animals were observed for mortality, clinical signs, body weight, food consumption, hematology, biochemistry and urinary parameters, pathology and toxicokinetics. Control animals received an injection of the vehicle solution. Blood samples were collected on each administration day (day 0 and day 7) at different time points such as 5 and $30 \mathrm{~min}$, and 1, 2, 6 and $24 \mathrm{~h}$ post injection.

Hematoxylin and eosin staining was performed on tissue sections excised from the heart, lung, kidneys and liver to visualize the toxicity induced by AGuIX NPs, 1 ( $n=5 /$ sex/group) or $10(n=3 /$ sex/group) weeks after the second and last injection.

\section{L Tumor rat model}

The 9L cells were isolated in the early 1970s by Benda et al. following repeated intravenous administration of $\mathrm{N}$-methyl-nitrosourea to Fischer rats ( $5 \mathrm{mg} / \mathrm{kg}$ weekly for 8 months) [26]. The so-called 9L-European Synchrotron Radiation Facility (ESRF) cells $[27,28]$ are derived from 9L cells acquired at the Brookhaven National Laboratory (NY, USA) in 1997. As previously described [27], 10-week-old Fischer 344 rats were anesthetized and placed on a stereotactic head holder. Then, $10^{4}$ 9L-ESRF viable cells suspended 
in $1 \mu \mathrm{l} \mathrm{DMEM}+1 \%$ penicillin/streptomycin were pushed manually within $30 \mathrm{~s}$ using a Hamilton syringe inserted into the right caudate nucleus $(9 \mathrm{~mm}$ anterior to the ear bars, in other words, at the bregma, $3.5 \mathrm{~mm}$ lateral to the midline, at $6 \mathrm{~mm}$ depth from the skull surface). All time intervals stated in this manuscript are post implantation, in other words, $\mathrm{D}_{10}$ means 10 days after tumor implantation.

Male Fischer rats were anesthetized by inhalation of $5 \%$ isoflurane in air followed by an intraperitoneal injection of xylazine/ketamine $64.5 / 5.4 \mathrm{mg} / \mathrm{kg}$ for the implantation procedure and irradiation, while they were maintained under isoflurane $2.5 \%$ in air for MRI examination combined with the NPs' injection. In any case, the eyes of the animals were embedded with a drop of Ocry-gel (TVM Laboratories, Lempdes, France) during the anesthesia to avoid dryness of the cornea. A synthesis of the history for each animal is given in Supplementary Table 2.

\section{Gd-based NPs}

The Gd-based AGuIX NPs were synthesized and characterized according to a previously described protocol (see Supplementary Figure 1) [13,29]. Briefly, the NPs are synthesized through a top-down process and are made of Gd chelates (1,4,7,10-tetraazacyclododecane1-glutaric acid-4,7,10-triacetic acid, i.e., DOTAGA) covalently linked to a polysiloxane network core. During synthesis, $\mathrm{pH}$ was adjusted to $7.2 \pm 0.15$ and the final hydrodynamic diameter was precisely controlled to reach $3 \pm 1 \mathrm{~nm}$, favoring renal elimination. AGuIXs were injected in the saphenous vein at a $100 \mathrm{~g} / \mathrm{l}$ concentration in a $1 \mathrm{ml}$ volume. The $\mathrm{r}_{1}$ value used for the MRI calculation was equal to $6 \mathrm{mM}^{-1} \cdot \mathrm{s}^{-1}(7 \mathrm{~T})$, while the $r_{2} / r_{1}$ ratio is $2.2(7 \mathrm{~T})$ (compared with 1.14 at 1.4 T) [13]. More than $90 \%$ of the injected NPs are washed out by the kidneys within the first day after injection, allowing a safe elimination [30].

\section{MRI acquisitions}

MRI was performed on a $7 \mathrm{~T}$ scanner (Biospec 70/20, Bruker Avance III console, Germany - Grenoble MRI facility IRMaGE) equipped with a $660 \mathrm{mT} / \mathrm{m}$ gradient coil and volume transmit/surface receive radiofrequency coils. Animals were placed in the prone position. All images were acquired in the coronal orientation. The MRI protocol was composed of three sequences:

Anatomical images ( $\mathrm{T}_{2}$-weighted) Rapid Imaging with Refocused Echoes (RARE)-T 2 MRI (repetition time (TR)/effective echo time (TE) 2500/40 $\mathrm{ms}$, number of average $(\mathrm{NA})=2$, slice thickness $=1$ $\mathrm{mm}$, reconstruction voxel size $=117 \times 117 \times 1000 \mu \mathrm{m}$, field of view $(\mathrm{FOV})=3 \times 3 \mathrm{~cm})$. This sequence was merely used for tumor localization and evaluation of the tumor volume;

$\mathrm{T}_{1}$-weighted spin-echo images (TR/TE 800/5 ms, $\mathrm{NA}=4$, slice thickness $=1 \mathrm{~mm}$, reconstruction voxel size $=234 \times 234 \times 1000 \mu \mathrm{m}, \mathrm{FOV}=3 \times 3 \mathrm{~cm}$ ). This sequence was acquired to assess the spatial extent of the extravasation of the contrast agent;

$\mathrm{T}_{1}$ maps (inversion recovery obtained with spinecho echo planar imaging (EPI) sequence and 18 inversion times values in the range [35-7200] $\mathrm{ms}, \mathrm{TR} /$ $\mathrm{TE}=18,000 / 20 \mathrm{~ms}, \mathrm{NA}=1$, slice thickness $=1 \mathrm{~mm}$, reconstruction voxel size $=234 \times 234 \times 1000 \mu \mathrm{m}$, FOV $=3 \times 3 \mathrm{~cm}$ ). This sequence was used to estimate the concentration in NPs in the tumor region.

The images from sequences I to III were obtained from the same imaging locations.

First, a preliminary study was performed over three rats at D14 stage in order to follow up the biodistribution of AGuIX NPs in brain tumor, the contralateral hemisphere and the cheek muscles. The rats were imaged as previously described $1,4,7$ and $24 \mathrm{~h}$ after injection of AGuIX NPs. Indeed the D14 stage guarantees a tumor mature enough to be visible in MRI while D10 as an earlier stage is more appropriate for radiotherapy [27].

Second, the presence and the size of the tumors of the 43 rats to be engaged in the imaging-guided radiotherapy experiment were quickly checked thanks to an anatomical $\mathrm{T}_{2}$-weighted sequence at $\mathrm{D} 10$.

The rats were then randomized in four groups: nontreated animals (NI, $\mathrm{n}=5$ ), control with AGuIX NPs injection (AGuIX-NI, $\mathrm{n}=6$ ), irradiation (RT, $\mathrm{n}=15)$ and irradiation after AGuIX NPs injection (AGuIX+RT, $\mathrm{n}=17)$. For the rats with an injection of AGuIX NPs, the MRI was performed using the sequences described previously. The rats were imaged and/or irradiated the same day at D10 and D17 following a single AGuIX NPs injection (Figure 1). The time elapsed between the injection of AGuIX NPs and the MRI acquisition was $2 \mathrm{~h} 25 \mathrm{~min}( \pm 8 \mathrm{~min}, \mathrm{n}=18)$, and $2 \mathrm{~h} 35 \mathrm{~min}( \pm 1 \mathrm{~min}, \mathrm{n}=18)$ at D10 and D17, respectively.

For the tumor volume analysis, as there is no significant difference ( $\mathrm{p}=0.273)$ between the two control groups (i.e., NI and AGuIX-NI), they were merged and called $\mathrm{NI}_{\mathrm{t}}$ group $(\mathrm{n}=11)$.

\section{MRI data processing \& analysis}

MRI data were processed in a Matlab environment (v7.6, The MathWorks Inc., MA, USA). Both the presence and the position of the tumor in MR images were assessed by two independent users for all the rats, according to a blinded procedure. For each MR image, tumors were identified and regions of inter- 
est (ROIs) in the tumor, in the contralateral brain and in the cheek muscle were manually delineated to measure the total average signal. The ROIs defined on the $\mathrm{T}_{2}$-weighted images were then extended to the others MRI sequences $\left(T_{1}\right.$-weighted and $T 1$ map images).

\section{Tumor volume}

The tumor volume was then estimated by adding the surfaces of each tumor section at D10 and D17.

\section{AGuIX concentration}

The $T_{1}$ maps were obtained by fitting, pixel by pixel, the inversion recovery data with a 3-parameter, exponential recovery, model [31]. The concentration of $\mathrm{Gd}^{3+}$ was calculated using the relaxation time determined on the $\mathrm{T}_{1}$ maps, according to the formula

$$
\left[G d^{3+}\right]=\frac{1}{r_{1}}\left(\frac{1}{T_{1}(t)}-\frac{1}{T_{1}(t 0)}\right)
$$

where $\mathrm{T}_{1}(\mathrm{t} 0)$ is the relaxation time before AGuIX injection (in seconds), $T_{1}(t)$ is the relaxation time at a determined time after NPs' injection (in seconds) and $r_{1}$ is the relaxivity per $\mathrm{Gd}^{3+}$ in AGuIX NPs at $7 \mathrm{~T}\left(6 \mathrm{mM}^{-}\right.$ $\left.{ }^{1} \cdot s^{-1}\right)[13]$.

\section{Statistical analyses}

Statistical analyses were performed using the two-tail nonparametric Mann-Whitney t-test. AGuIX concentrations were expressed as the mean \pm standard deviation (SD). The tumor volumes and the mean survival times (MST) were expressed as the mean \pm standard error to the mean (SEM). The survival data were analyzed using a log-rank test. In both cases, statistical significance was considered when $\mathrm{p}<0.05$.

\section{Radiotherapy}

Irradiations were performed at the Grenoble Alpes University Hospital using a 6-MV medical irradiator (SLI, Elekta, Crawley, UK). Rats were irradiated, six at a time (see Figure 2) in a customized immobilization device including a $2-\mathrm{cm}$ tissue equivalent bolus to ensure the electronic equilibrium. Dosimetry and delineation were performed using an Eclipse ${ }^{\mathrm{TM}}$ treatment planning system (Varian, NY, USA) on a dosimetry computed tomography (CT) scanner (General Electric Healthcare, UK).

A fusion between CT scan and MRI was performed on one rat to determine the doses received by the tumor and organs at risk (mainly eyes and spinal cord). Rats were treated by a monoisocentric conformational technique using one anterior and one posterior field without filter. The dose prescription was two fractions of 10 Gy at D10 and D17 after tumor implantation. The time elapsed between the injection of the AGuIX NPs and the radiation exposure was $7 \mathrm{~h}$ $33 \mathrm{~min}( \pm 38 \mathrm{~min}, \mathrm{n}=17)$ and $6 \mathrm{~h} 57 \mathrm{~min}( \pm 27 \mathrm{~min}$, $\mathrm{n}=17)$ at D10 and D17, respectively. The survival rats were lastly imaged at D32 when possible $(n=14 / 30)$.

\section{Survival analysis}

The rats were followed at the animal facility after the radiotherapy and they were euthanized by intracardiac injection of pentobarbital sodium less than 1 day before their anticipated death as judged by clinical signs, except when found dead. The time between implantation and death was recorded as survival time (one day was added for euthanized rats) and plotted. The MST \pm SEM and the increase in life span (ILS) were calculated (Prism, GraphPad Software, V6.2, CA, USA). The ILS is equal to the difference between

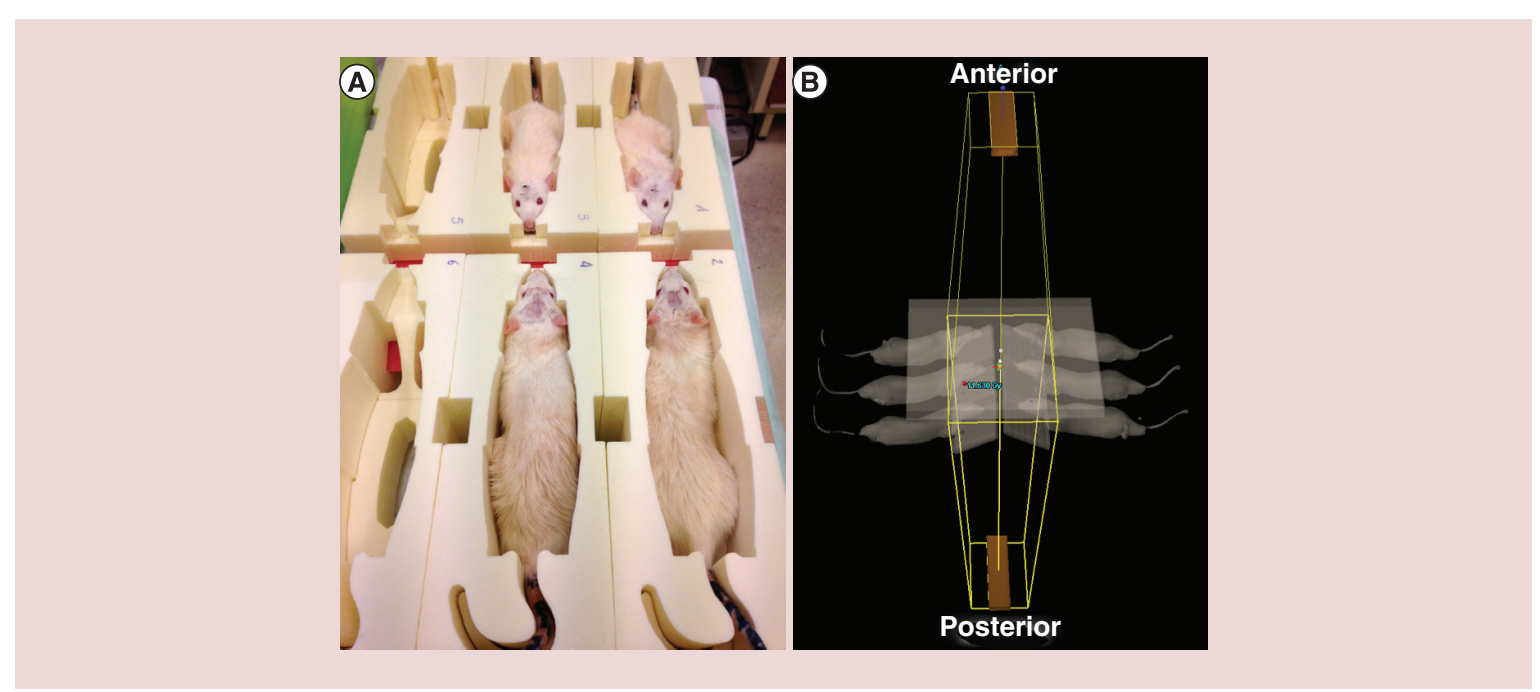

Figure 2. Experimental setup used for in vivo radiation therapy of the rats. (A) Six rats were immobilized and irradiated simultaneously. (B) Anterior and posterior 6-MV fields for whole brain radiation therapy. 


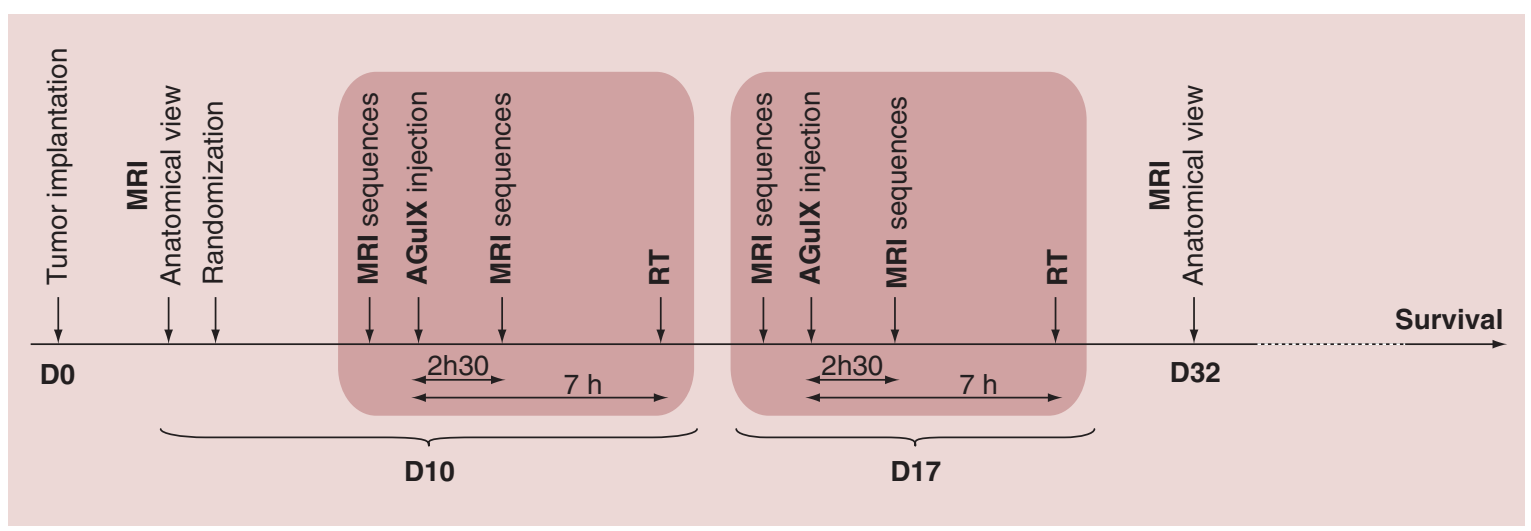

Figure 1. Diagram of the imaging and radiotherapy experimental protocol.

the MST for treated and untreated rats divided by the MST for untreated rats.

\section{Histology}

The brain of each euthanized rat was rapidly sampled, frozen in precooled isopentane at $-50^{\circ} \mathrm{C}$ and stored at $-80^{\circ} \mathrm{C}$. Horizontal brain sections $(18 \mu \mathrm{m})$ were cut at $-20^{\circ} \mathrm{C}$ on a cryostat (Microm HM 80, Thermo Fisher Scientific, France). A standard Masson's trichrome staining was performed in those sections where the tumor appeared the largest. A double-blind qualitative analysis of the slides was performed.

\section{Results}

Toxicity evaluation \& pharmacokinetics

Good laboratory practices regulatory toxicology and pharmacokinetics were evaluated in rats after two repeated intravenous administrations of AGuIX NPs at a 1-week interval. The rats presented absolutely no modification of any observed antemortem parameters at any dose (see Supplementary Table 1). At the end of the first week or tenth week observation period, no AGuIX-related effects on organ weights or macroscopic organ changes were noted (Figure 3). The only histopathological change was a dose-dependent minimal multifocal vacuolation of the corticotubular epithelium of the kidney, after the 1-week observation period. This feature was also observed in mice in previous studies [30] and was considered to be nonadverse. Indeed, after the 10-week recovery period, only one single female previously treated twice at the high dose of $750 \mathrm{mg} / \mathrm{kg}$ still presented some weak vacuolations (Figure 3, bottom panel), indicating almost complete reversibility under the conditions of the study. In all

Table 1. Mean pharmacokinetic parameters in male and female Wistar rats following two intravenous administration of AGuIX nanoparticles.

\begin{tabular}{|c|c|c|c|c|c|c|c|c|c|c|c|}
\hline Day & Gender & $\begin{array}{l}\text { Dose (mg/ } \\
\text { kg/day) }\end{array}$ & $\begin{array}{l}A^{A U C_{i n f}} \\
\text { (h.ng/ml) }\end{array}$ & $\begin{array}{l}\text { DN } \\
\text { AUC }_{\text {inf }}\end{array}$ & $C_{0}(n g / m l)$ & $C_{\max }(\mathrm{ng} / \mathrm{ml})$ & $D N C_{\max }$ & $T_{1 / 2}(h)$ & $\begin{array}{l}\mathrm{Cl}(\mathrm{I} / \mathrm{h} / \\
\mathrm{kg})\end{array}$ & $\mathrm{V}_{\mathrm{ss}}(\mathrm{I} / \mathrm{kg})$ & $\begin{array}{l}\text { Acc. } \\
\text { ratio }\end{array}$ \\
\hline Day 0 & Male & 250 & 429,000 & 1720 & $1,050,000$ & 780,000 & 3120 & 0.83 & 0.583 & 0.311 & - \\
\hline Day 0 & Male & 750 & $1,340,000$ & 1790 & $2,820,000$ & $2,160,000$ & 2880 & 2.55 & 0.559 & 0.487 & - \\
\hline Day 0 & Female & 250 & 482,000 & 1930 & $1,120,000$ & 830,000 & 3320 & 1.19 & 0.519 & 0.374 & - \\
\hline Day 0 & Female & 750 & $1,300,000$ & 1730 & $2,870,000$ & $2,200,000$ & 2940 & 2.63 & 0.578 & 0.505 & - \\
\hline Day 7 & Male & 250 & 462,000 & 1850 & $1,070,000$ & 814,000 & 3260 & 2.62 & 0.541 & 0.398 & 1.08 \\
\hline Day 7 & Male & 500 & 805,000 & 1610 & $1,520,000$ & $1,240,000$ & 2480 & 3.04 & 0.621 & 0.508 & 0.967 \\
\hline Day 7 & Male & 750 & $1,420,000$ & 1890 & $3,240,000$ & $2,430,000$ & 3250 & 2.81 & 0.528 & 0.455 & 1.06 \\
\hline Day 7 & Female & 250 & 483,000 & 1930 & $1,150,000$ & 832,000 & 3330 & 3.03 & 0.518 & 0.529 & 1.01 \\
\hline
\end{tabular}


dose groups, there was no indication of degeneration or necrosis of renal epithelial cells.

The pharmacokinetic parameters of AGuIX NPs were summarized in Table 1. Blood exposure increased in a dose-proportional manner for both sexes on each evaluation day. Accumulation ratios ranged from 0.916 to 1.08 at all dose levels, meaning that basically there is no accumulation of AGuIX NPs.

Mean clearance and volume of distribution were low and ranged from 0.518 to $0.631 \mathrm{l} / \mathrm{h} / \mathrm{kg}$ and 0.311 to $0.529 \mathrm{l} / \mathrm{kg}$, respectively. Mean $\mathrm{T}_{1 / 2}$ ranged from 0.834 to $3.04 \mathrm{~h}$. In general, there was no difference related to dose, sex or evaluation days for clearance, volume of distribution or $T_{1 / 2}$ values.

Based on the absence of overt evidence of toxicity, after two repeated intravenous administrations, the highest tested dose $(750 \mathrm{mg} / \mathrm{kg} / \mathrm{administration})$ was considered to be the nonobserved adverse effect level. This dose corresponds to an area under the concentration-time curve from zero up to the infinity of
$1340 / 1420 \mu \mathrm{g} . \mathrm{h} / \mathrm{ml}$ (day 0/day 7) in males and of $1300 / 1190 \mu \mathrm{g} . \mathrm{h} / \mathrm{ml}$ (day 0/day 7) in females.

\section{Follow-up of AGulX NPs kinetic by MRI}

The AGuIX NPs' distribution was followed up at D14 after tumor implantation to allow MRI measurements. Although the tumors were not visible on $\mathrm{T}_{1}$-weighted images acquired before the intravenous injection of AGuIX NPs, a very marked positive contrast enhancement of the outer part of the tumor was observed $1 \mathrm{~h}$ after the injection of AGuIX NPs (Figure 4A). After this first step of tumor accumulation, the images were less and less contrasted indicating a washout of the NPs from the tumor. However, as a major outcome, the tumor contrast was still visible $24 \mathrm{~h}$ after injection, despite a gradually decrease of the signal over time, for both the tumor, and the surrounding tissues (Figure 4A).

Using the acquisition of $T_{1}$ maps, and as shown in Figure $4 \mathrm{~B}$, the concentration of $\mathrm{Gd}^{3+}$ in AGuIX NPs

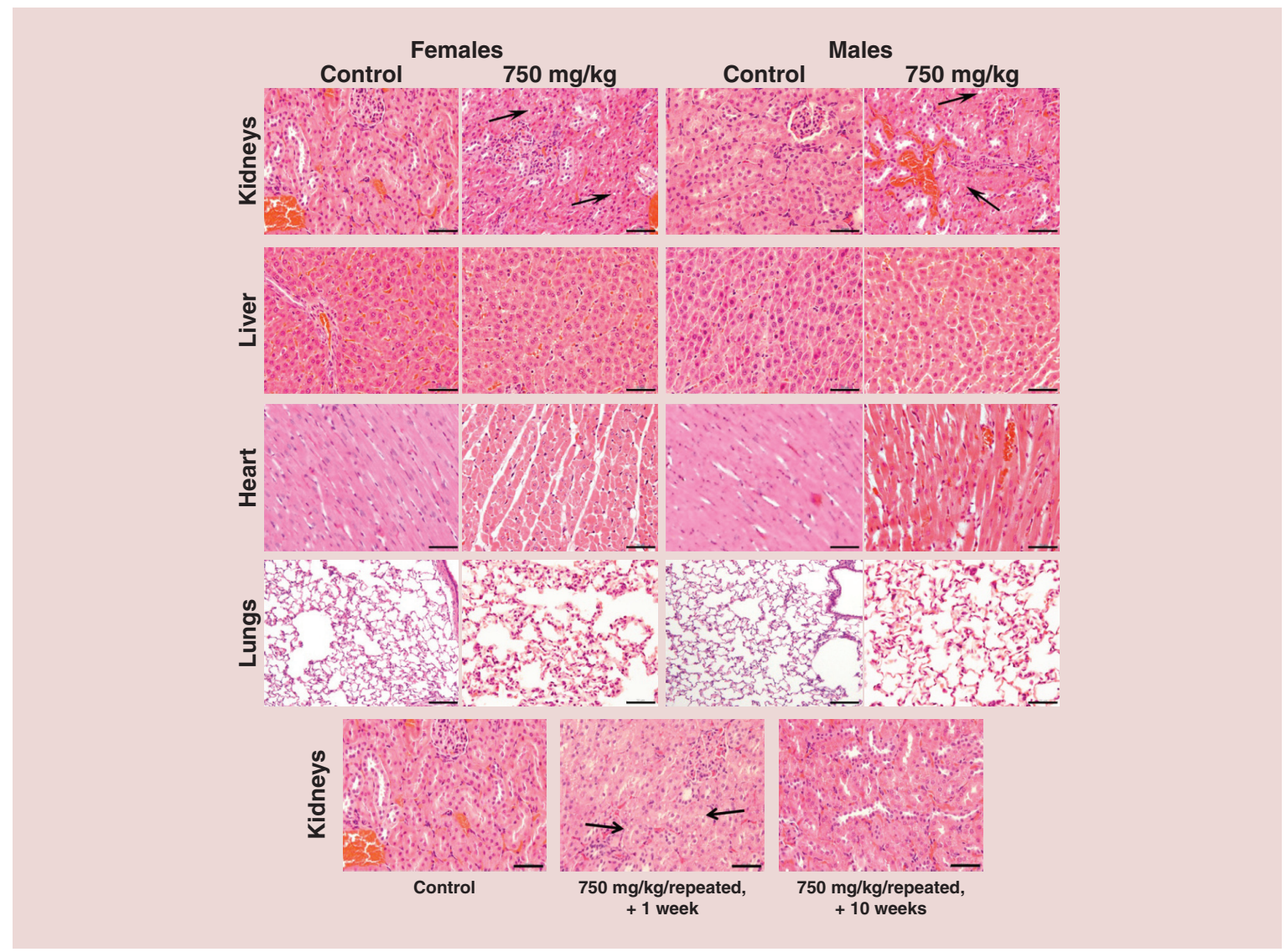

Figure 3. Hematoxylin and eosin staining of histological sections of vital organs and injection site. No microscopic changes in all the tissues were evidenced after two administrations of AGuIX NPs as compared with the control group, both in males and females, except for kidneys. Minimal vacuolation of the corticotubular epithelium could be observed 1 week after administration in all groups (black arrows). After a 10-week recovery period, very weak vacuolation was detected only for one female, as presented in the bottom right panel. This recovery period allowed to observed kidneys with similar microscopic aspect as compared with control condition, for all the other animals at any dose. Scale bar: $50 \mu \mathrm{m}$. 


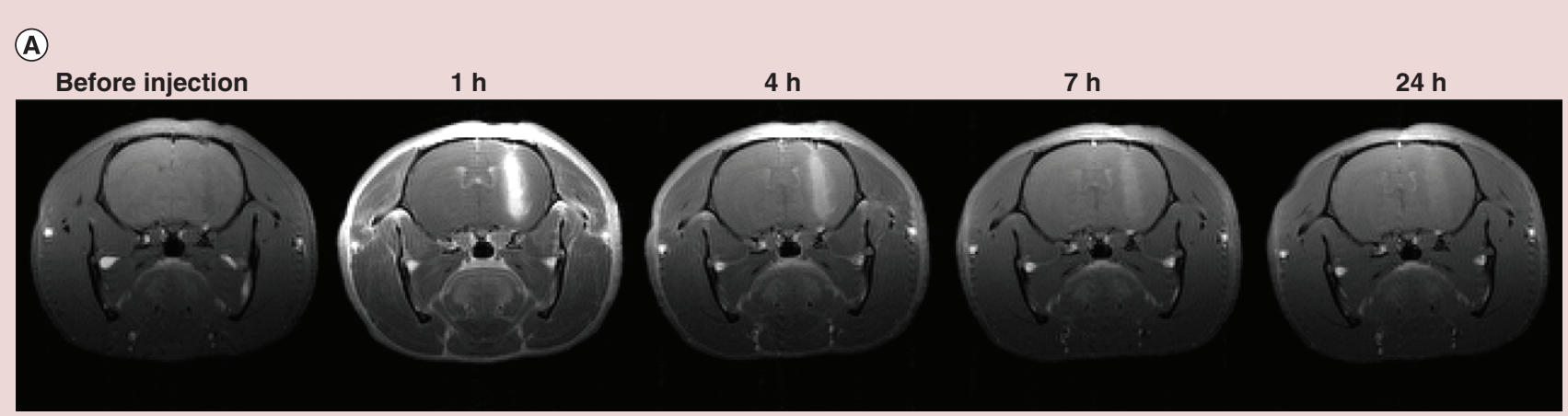

(B)

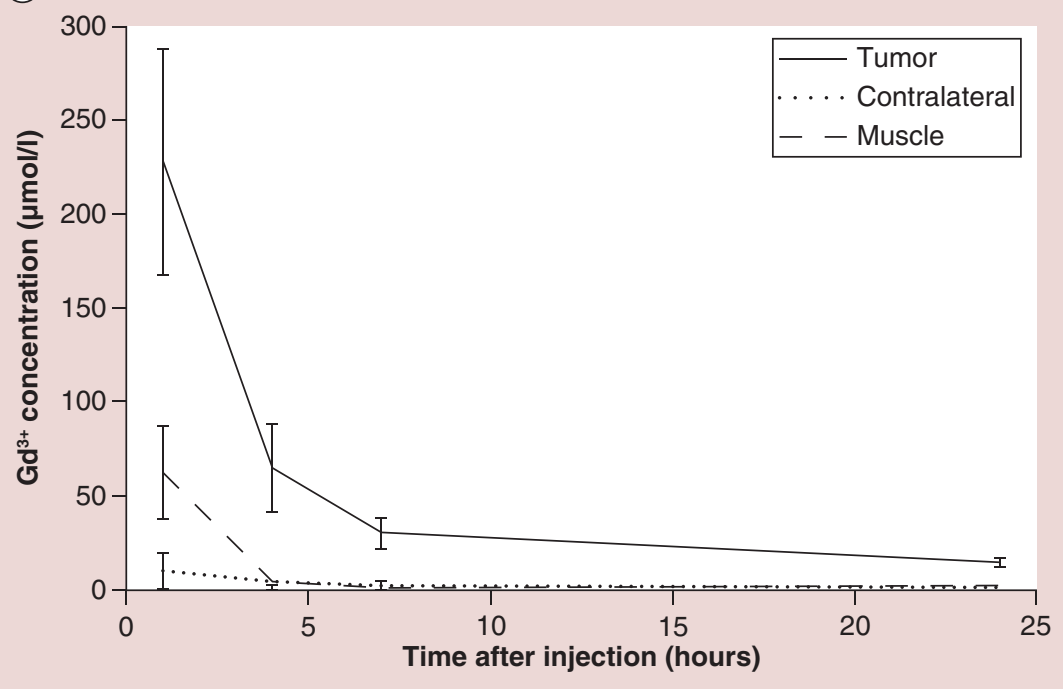

Figure 4. MRI and gadolinium quantification in 9L-ESRF-bearing rats. (A) The $T_{1}$-weighted MR images were acquired before and $1,4,7$ and $24 \mathrm{~h}$ after intravenous injection of $1 \mathrm{ml}$ of AGulX NPs $\left(\left[\mathrm{Gd}^{3+}\right]=100 \mathrm{mM}\right)$ over three rats at D14. The pictures indicate an enhancement of tumor- $\mathrm{T}_{1}$ contrast due to the Gd-based nanoparticles, until $24 \mathrm{~h}$ post intravenous injection. (B) Gadolinium concentrations issued from $T_{1}$ maps as a function of time elapsed after injection $(n=3)$ in three regions of interest. The results were expressed as the means \pm standard deviation.

was determined, and the quantitative results obtained perfectly fit with the qualitative analysis presented in Figure 4A. Especially $1 \mathrm{~h}$ after administration, the concentration of $\mathrm{Gd}^{3+}$ in AGuIX NPs achieved a maximum in the tumor, reaching $227.9 \pm 60 \mu \mathrm{M}$ in the tumor against $10.5 \pm 9.2$ and $62.9 \pm 24.7 \mu \mathrm{M}$ in the contralateral area (ratio 21.62) and the cheek muscle (ratio 3.62), respectively. Then the values gradually decreased in the three tissues of interest, with a slower washout in the tumor region.

In parallel, the concentration of $\mathrm{Gd}^{3+}$ in the muscle rapidly decreased with values from $62.9 \pm 24.7,5 \pm 2.6$, $0.6 \pm 1.1$ and $0.8 \pm 1.4 \mu \mathrm{M}$ at $1,4,7$ and $24 \mathrm{~h}$, respectively, reflecting the elimination of the NPs that were not retained by the tumor, elimination which occurred by the renal route $[14,30]$.

Finally, the tumor-to-contralateral (T/C) ratios of AGuIX NPs concentration decreased from $21.621 \mathrm{~h}$ after injection to 18.12 at $4 \mathrm{~h}, 14.52$ at $7 \mathrm{~h}$, and 16 at 24 $\mathrm{h}$ after injection. Especially $7 \mathrm{~h}$ after injection, the T/C ratio was still high, and favorable for a selective treatment of tumors. Remarkably, at 24 h, the AGuIX NPs' concentration was weak in the tumor region, but the T/C ratio was still advantageous. These results indicated a favorable $\mathrm{T} / \mathrm{C}$ ratio for radiosensitization, in other words, a large therapeutic window for radiation exposure.

\section{Dosimetry calculations \& measurements before radiotherapy}

For MRI and radiation therapy, the animals were divided into four groups as indicated in the 'Materials \& methods' section (see Supplementary Table 2). Similar to clinical practices, tumor and organs at risk were delineated before radiation exposure based on MR and CT fusion. Because of very small tumor volumes $(<0.1$ $\mathrm{cm}^{3}$ ), whole brain was chosen as the clinical target volume. The dose was delivered with two opposite anterior and posterior beams, with the isocenter at the center of the brain. The calculated dose indicated that the whole brain received a total mean dose of 11.3 Gy. Sparing 


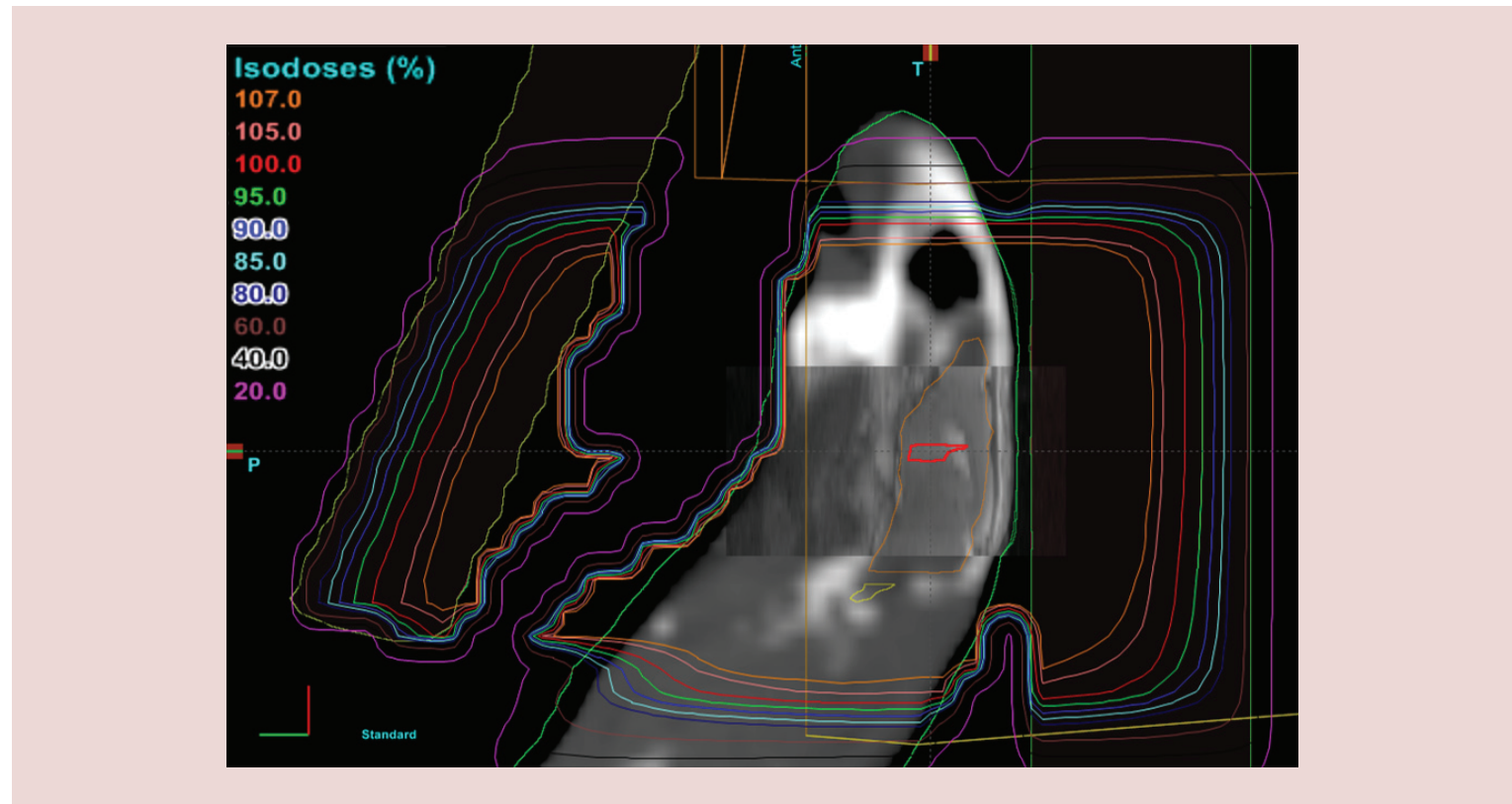

Figure 5. Dosimetric isodoses representation on computed tomography scan-MRI fusion. Gross tumor volume: macroscopic tumor delineated in red; clinical target volume: whole brain delineated in orange.

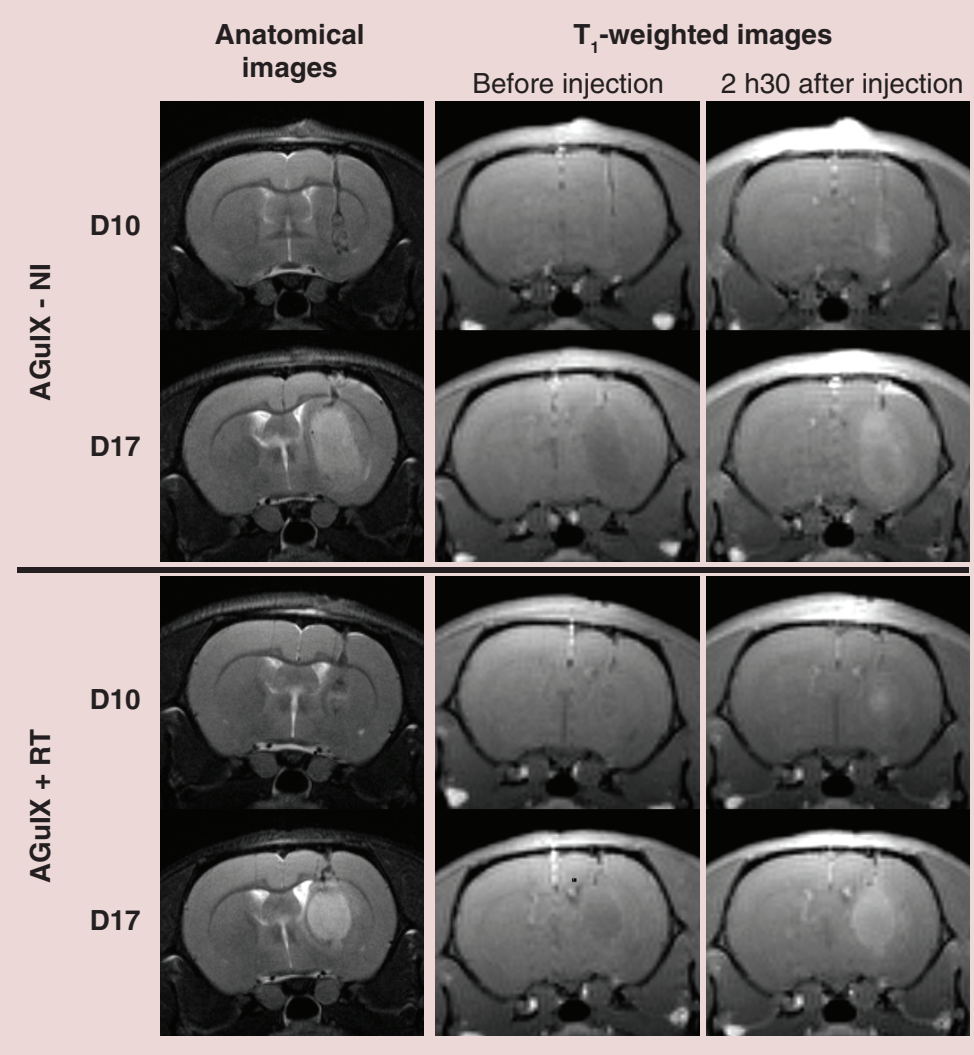

Figure 6. $T_{2}$ - and $T_{1}$-weighted MR images of 9L-ESRF-bearing rats. The images were acquired at D10 and D17, before and $2 \mathrm{~h} 30 \mathrm{~min}$ after intravenous injection of AGuIX NPs. The anatomical views ( $\mathrm{T}_{2}$-weighted images) allowed the localization of the tumors. The $T_{1}$-weighted images illustrated the tumor contrast enhancement after the intravenous injection of AGuIX NPs. 


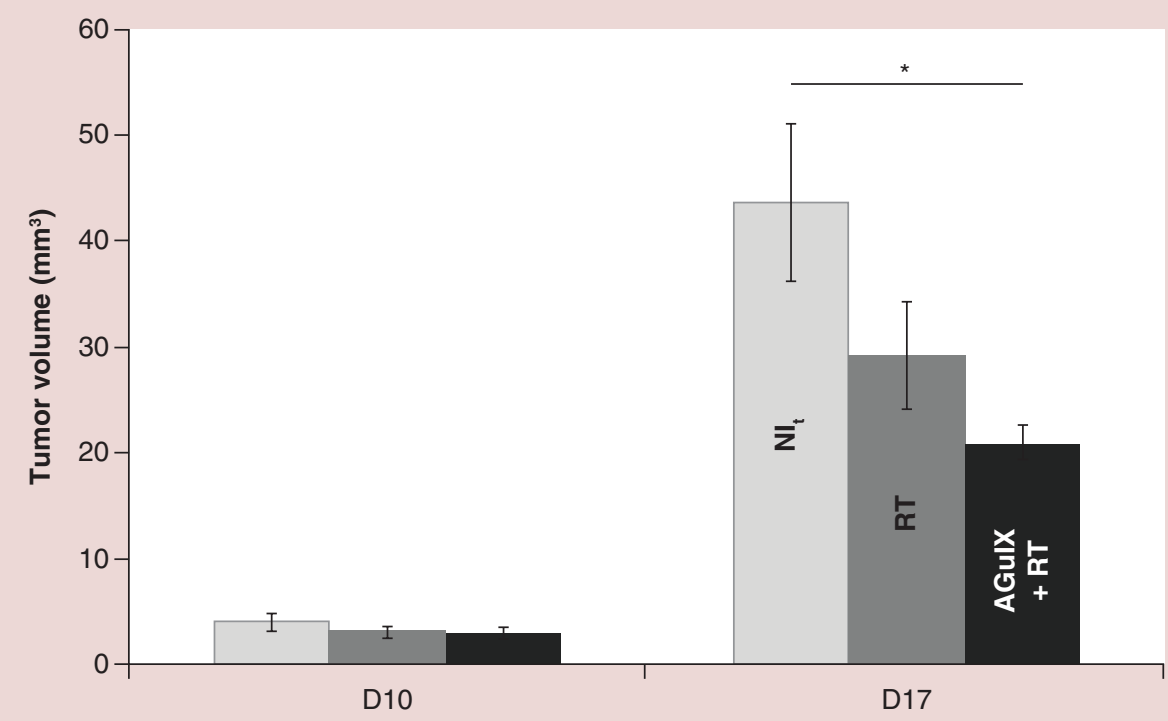

Figure 7. Tumor volume measured at D10 and D17, just before each radiotherapy session for non irradiated animals ( $\left.\mathrm{NI}_{\mathrm{t}}, \mathrm{n}=11\right)$, irradiated animals without particle injection $(\mathrm{RT}, \mathrm{n}=15)$ and irradiated animals after AGuIX NPs' injection (AGuIX+RT, $\mathbf{n}=14$ ). The results are expressed as the mean \pm standard error of the mean. RT: Radiation therapy.

eyes balls were not possible with a mean dose to the eyes at $11.3 \mathrm{~Gy}$. The mean dose to the heart, lung and spinal cord was $0.3,0.3$ and $2 \mathrm{~Gy}$, respectively (Figure 5).

MRI follow-up of tumor volume evolution \& contrast enhancement during the treatment Based on the anatomical MR images performed at $\mathrm{D} 10$, the rats were homogeneously randomized in the different groups (mean tumor volume \pm SEM; NI: $3.97 \pm 0.87$, RT: $3.10 \pm 0.52$, AGuIX+RT: $2.85 \pm 0.61$ $\mathrm{mm}^{3}$, respectively).

Although smaller in size and less vascularized as compared with D14, the tumors were visible at D10 and appeared with a positive contrast enhancement after AGuIX NPs' injection. The tumors exhibited roughly the same contrast uptake for both irradiated and nonirradiated animals at D17 as compared with D10 (Figure 6).

Furthermore, at D17 (i.e., 7 days after the first irradiation exposure), significant differences were found between the control group and the irradiated groups alone and combined with the injection of AGuIX NPs ( $\mathrm{p}=0.021$ and $\mathrm{p}=0.0037$, respectively), with mean tumor volumes equal to $47.15 \pm 7.25,29.18 \pm 5.09$, $21.78 \pm 2.08 \mathrm{~mm}^{3}$, respectively (Figure 7).

\section{Survival of the animals}

The survival was observed after treatment for the different groups (Figure 8). The MST was equal to 26 \pm 0.5 days for the controls animals and $39 \pm 2$ days for the irradiated animals. By combining the radio- therapy and AGuIX NPs, the MST increased up to $72.9 \pm 35.5$ days. Consequently, the ILS increased up to $134 \%$ by combining the radiotherapy with AGuIX NPs preinjection, against $50 \%$ for the radiotherapy alone. Remarkably, a long-term survivor had a tremendous lifespan, willingly stopped at day 533. While the tumor of this animal was very visible at D17 and D31, it has totally disappeared at D64, with no recurrence at D276 and D533 (Figure 9), as attested by the histology examination.

\section{Histology}

The tumor recurrence was observed for all animals, except for the long-term survival rat (Figure 9). At the end of life, the unirradiated tumors appeared histologically as a mostly well-limited mass invading the right hemisphere. Few figures of mitosis were found on each slice. Small islets of necrosis were found, mostly at the tumor center. Around these necrotic areas and until the external rim of the tumor, the density of tumor cells was high including cells with numerous atypic nuclei.

The irradiated tumors exhibited the same size than the unirradiated tumors at the end of the survival, as well as the same pattern in general. However, larger necrotic areas were observed instead of islets. Also the density of atypic nuclei in the tumor cell population was higher. No difference was seen at histology between the two irradiated groups (with and without AGuIX NPs). Moreover, there was no visible AGuIXrelated effect in the healthy part of the brain, as compared with control animals. 


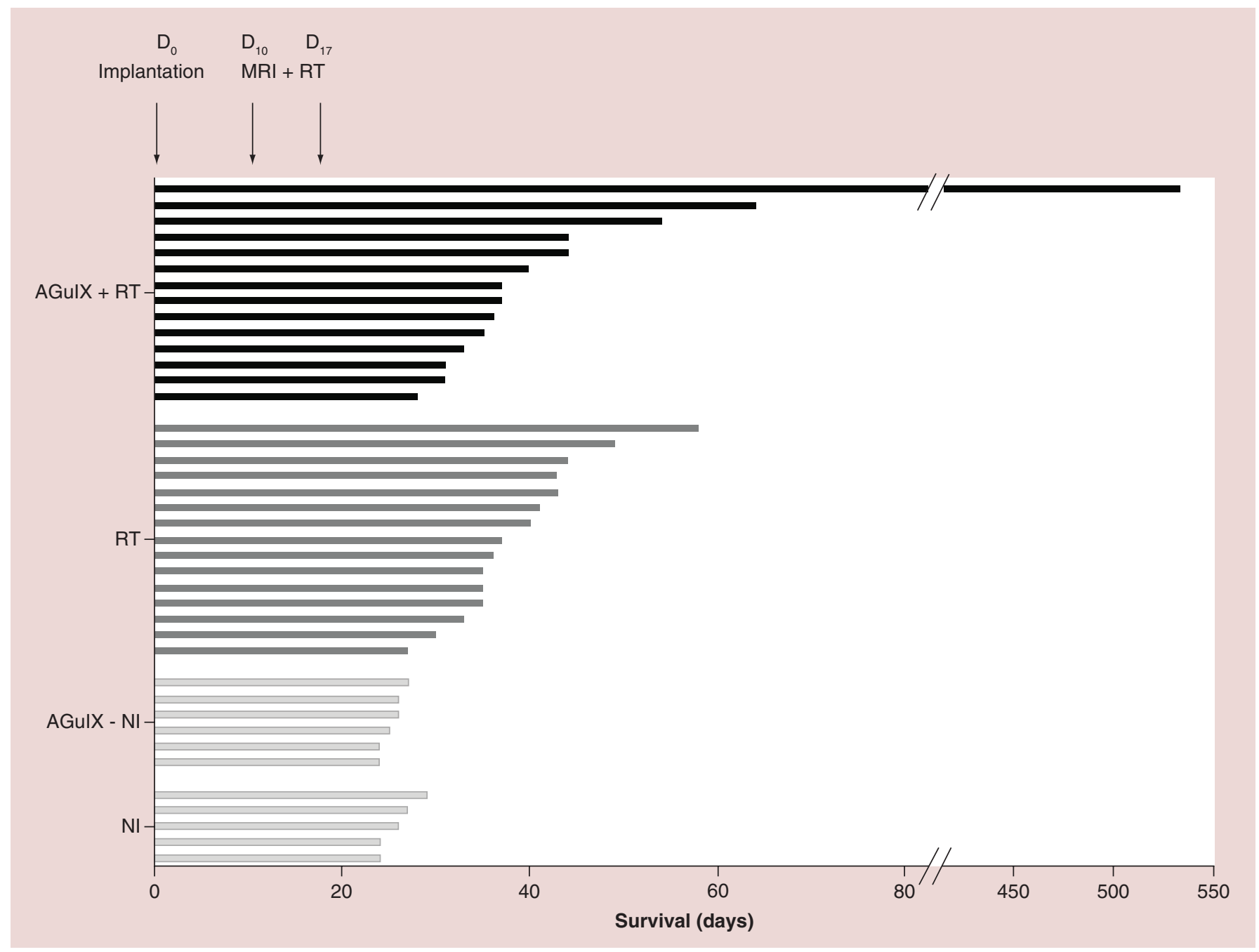

Figure 8. Schematic representation of the survival of 9L-ESRF-bearing rats for the different treatments (NI, $n=5$; AGuIX-NI, $n=6$; RT, $\mathrm{n}=15$; AGuIX+RT, $\mathbf{n}=14)$. The length of the lines represents the survival time of rats after tumor implantation.

AGulX-NI: Control with AGuIX NPs injection; RT: Radiation therapy.

\section{Discussion}

While few NPs containing high-Z elements are currently being evaluated in vitro and in vivo in various academic laboratories worldwide, there are currently no clinical trials in progress delivering NPs intravenously for radiosensitization. The purpose of this study was to establish a preclinical proof of concept for MRI-guided AGuIX radiosensitization on rats bearing intracerebral glioma, using a clinical 6-MV irradiator.

The MRI data demonstrate a strong tumor uptake after intravenous administration of the NPs since the first hour after injection, along with a tumor retention up to $24 \mathrm{~h}$. As previously reported [14,22], such $\mathrm{T}_{1}$ contrast enhancement is visible for a shorter duration in the case of molecular Gd compounds, such as Dota$\mathrm{rem}^{\circledR}$ which was totally washed out within the first hour after intravenous injection. The clustering of Gd chelates within the small AGuIX NPs allows an effi- cient tumor retention while maintaining a rapid renal clearance.

The persistence of a favorable $\mathrm{T} / \mathrm{C}$ ratio as a function of time suggests that a single AGuIX NPs' injection may allow us to maximize the local dose during two radiation exposures. On the safe side, radiation exposure might be performed after the first blood half-life (i.e., $2 \mathrm{~h}$ in rats), to reduce the amount of AGuIX NPs still present in healthy tissues. Besides, early imaging appeared promising for precise tumor delineation, as previously demonstrated [23,32]. In the case of glioma, as well as in any vascularized tumors, AGuIX NPs are able to leak from the tumor-induced disrupted vasculature, while they cannot extravasate in the healthy surrounding tissue. The high tumor retention and the longer blood half-life than molecular compounds, combined with the renal elimination, are crucial to maximize treatment efficacy while limiting side effects 


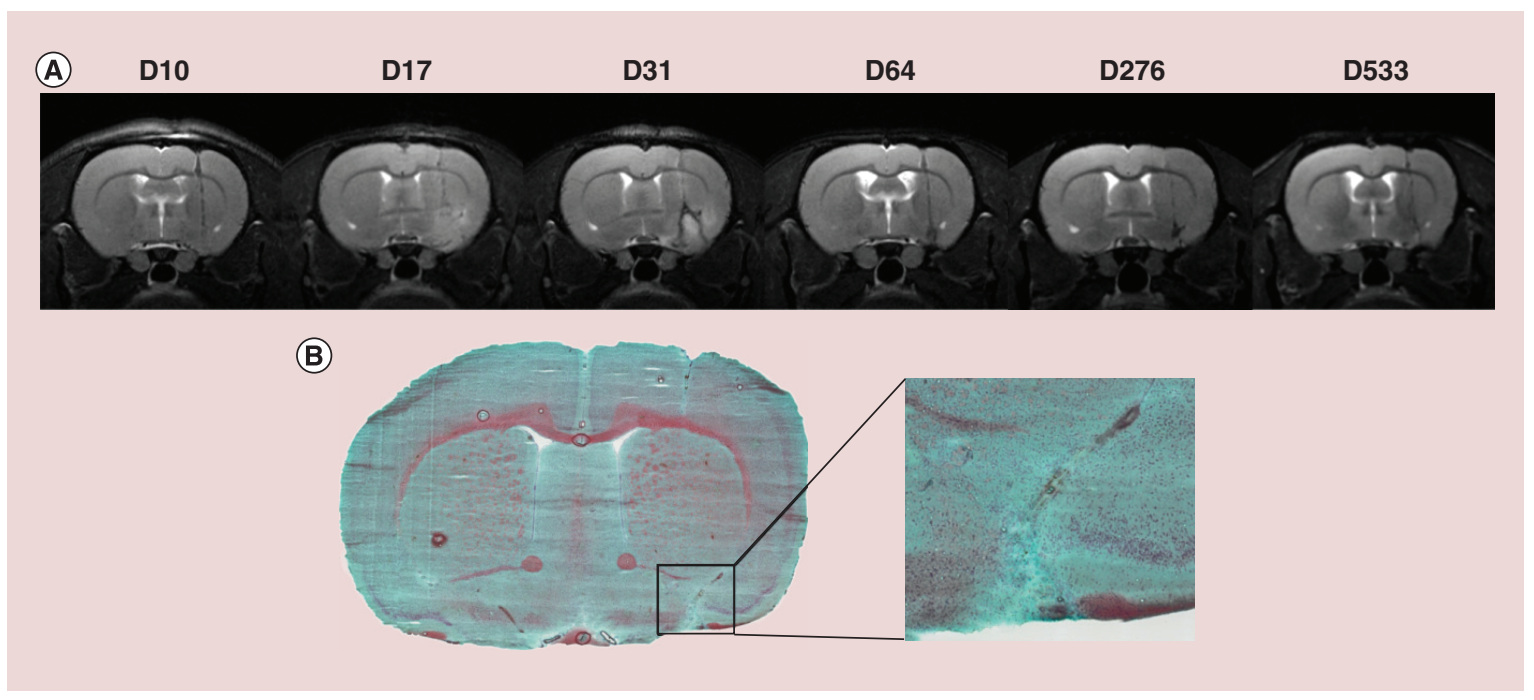

Figure 9. MRI and histological images of the long-term survivor rat. This animal was injected with AGuIX NPs before radiation exposure. (A) Anatomical $T_{2}$-weighted images. (B) Masson's trichrome staining of the brain at D533.

and toxicity. Moreover, AGuIX-induced radiosensitization allowed an increase of the radiation exposure efficacy, with a tumor volume reduction of $26 \%$ compared with RT group at D17. In addition, no evidence of toxicity at normal injection doses has been detected. Only transient alterations on the kidneys have been observed after high repeated injection in rodent; this phenomenon was not observed in nonhuman primates.

This study demonstrates the translational potential for MRI-guided radiation therapy. However, the optimization of both the dose and the fractionation is restricted using such an animal model. First, the current irradiator systems dedicated to humans do not allow to strictly restricting the delivered dose to the tumor in rodent models, explaining the choice for a pan-encephalic irradiation. Consequently, the high delivered dose could induce some toxicity in nontargeted areas, impairing with the survival. As an example, a 50\% end point value of $23 \mathrm{~Gy}$ at 39 weeks and $21 \mathrm{~Gy}$ at 52 weeks was reported for a 10 -mm radius field of irradiation (half of the rat brain) [33,34]. Finally, the clinical hypofractionation (2- or 3-Gy sessions) is not realistic on such fast growing rodent pathology. The progresses in terms of precision and tumor delineation are constant in radiotherapy, while sparing the healthy tissues. However, the enhancement of the focused dose due to NPs represents a strong conceptual breakthrough: a dose gradient might be delivered at the millimetric scale. In this case, the dose enhancement will not be affected by the patient repositioning that limits the external radiotherapy. The combination of NPs such as AGuIX with radiotherapy will thus be similar to internal radiation therapy, without the limitations of the use of radioactive compounds. Moreover, the activation of the AGuIX NPs will be controlled and performed only in the targeted ROI and at the appropriate moment.

We anticipated some variations in human tumor retention of AGuIX NPs depending on the tumor histology and grading, the tumor volume and the presence of necrotic regions. The imaging properties of the AGuIX NPs will permit to determine the better therapeutic protocol for each patient. The optimization of the combination 'AGuIX and radiotherapy' will be based on the data obtained during the first clinical trials.

\section{Conclusion \& future perspective}

This study has been performed to anticipate and design the first clinical trial with the AGuIX NPs: a single intravenous injection of AGuIX NPs will allow the MRI tumor contrast enhancement measurement before radiation exposure, and later on the long-term follow-up of patients, as a personalized treatment. Beyond brain tumors, this will pave the way for using AGuIX NPs as a generalist radiosensitizer drug in the treatment of cancers, in order to stimulate the dose effect in radiotherapy, without changing medical practices, in other words, intravenous injection complementary to chemotherapy if any.

\section{Supplementary data}

To view the supplementary data that accompany this paper please visit the journal website at: www.future-science.com/ doi/full/10.2217/nnm-2016-0203

\section{Financial \& competing interests disclosure}

This project was partially granted by Nano-Hand by two ANR projects (ANR-11-NANO-017 TheraGuIMA and ANR-12-RPIB-0010 MULTIMAGE). The Grenoble MRI facility IRMaGE was partly funded by the French program 'Investissement d'Avenir' run by 
the 'Agence Nationale pour la Recherche'; grant 'Infrastructure d'avenir en Biologie Santé' - ANR-11-INBS-0006. F Lux and O Tillement have to disclose the patent WO2011/135101. G Le Duc and O Tillement have to disclose the patent WO2009/053644. These patents protect the nanoparticles described in this publication: AGuIX ${ }^{\circledR}$. S Dufort is an employee from Nano-H SAS that is developing the AGuIX nanoparticles. S Dufort and G Le Duc are employees of NH TherAguix SAS that is developing the clinical applications of the AGulX nanoparticles. The authors have no other relevant affiliations or financial involvement with any organization or entity with a financial interest in or financial conflict with the subject matter or materials discussed in the manuscript apart from those disclosed.

No writing assistance was utilized in the production of this manuscript.

\section{Acknowledgements}

The experiment would not have been possible without the support of the platforms engaged. For this reason we gratefully thank the ESRF BMF staff (H Bernard, G Petre and D Colliat), the Charles River Laboratories staff (C Caloud), the CHU Unit radiotherapy staff members ( $R$ Philibert and $R$ Sihanath), the Grenoble MRI facility IRMaGE.

Ethical conduct of research

The authors state that they have obtained appropriate institutional review board approval or have followed the principles outlined in the Declaration of Helsinki for all human or animal experimental investigations. In addition, for investigations involving human subjects, informed consent has been obtained from the participants involved.

\section{Executive summary}

\section{Objective}

- To establish a preclinical proof of concept for MRI-guided AGuIX radiosensitization on rats bearing intracerebral glioma using a clinical 6-MV irradiator, after a unique intravenous (IV) administration of nanoparticles (NPs).

\section{Results}

- A strong tumor uptake was demonstrated by MRI since the first hour after intravenous injection of NPs, along with a tumor retention up to $24 \mathrm{~h}$.

- The persistence of a favorable tumor/contralateral ratio as a function of time suggests that a single AGuIX NP injection may allow a radiosensitizing effect during several daily radiation exposures.

- AGuIX NPs allow an increase of the radiation exposure efficacy, with a significant tumor volume reduction, after clinical 6-MV irradiation.

- Pharmacokinetics and regulatory toxicology studies demonstrated the safety profile of the AGuIX NPs, after two repeated IV administration of high doses of NPs and enabled the determination of the nonobserved adverse effect level in rodents (i.e., $750 \mathrm{mg} / \mathrm{kg} / \mathrm{administration).}$

- AGuIX NPs are activable NPs and exert their therapeutic activity only under irradiation.

\section{Conclusion}

- Both the efficacy and the translational potential of AGuIX NPs for image-guided radiation therapy were demonstrated, promising properties that will be assessed for clinical use in the upcoming Phase I clinical trial.

\section{References}

Papers of special note have been highlighted as: • of interest; •• of considerable interest

1 Stupp R, Hegi ME, Mason WP et al. Effects of radiotherapy with concomitant and adjuvant temozolomide versus radiotherapy alone on survival in glioblastoma in a randomised Phase III study: 5-year analysis of the EORTCNCIC trial. Lancet Oncol. 10(5), 459-466 (2009).

2 Canyilmaz E, Uslu GD, Colak F et al. Comparison of dose distributions hippocampus in high grade gliomas irradiation with linac-based imrt and volumetric arc therapy: a dosimetric study. SpringerPlus 4, 114 (2015).

3 Shaffer R, Nichol AM, Vollans E et al. A comparison of volumetric modulated arc therapy and conventional intensity-modulated radiotherapy for frontal and temporal high-grade gliomas. Int. J. Radiat. Oncol. Biol. Phys. 76(4), 1177-1184 (2010).

4 Brauer-Krisch E, Serduc R, Siegbahn EA et al. Effects of pulsed, spatially fractionated, microscopic synchrotron $\mathrm{x}$-ray beams on normal and tumoral brain tissue. Mutat. Res. 704(1-3), 160-166 (2010).

5 Kobayashi K, Usami N, Porcel E, Lacombe S, Le Sech C. Enhancement of radiation effect by heavy elements. Mutat. Res. 704(1-3), 123-131 (2010).

6 Mcmahon SJ, Mendenhall MH, Jain S, Currell F. Radiotherapy in the presence of contrast agents: a general figure of merit and its application to gold nanoparticles. Phys. Med. Biol. 53(20), 5635-5651 (2008).

7 Pradhan AK, Nahar SN, Montenegro M et al. Resonant $\mathrm{x}$-ray enhancement of the Auger effect in high- $Z$ atoms, molecules, and nanoparticles: potential biomedical applications. J. Phys. Chem. A 113(45), 12356-12363 (2009).

8 Her S, Jaffray DA, Allen C. Gold nanoparticles for applications in cancer radiotherapy: mechanisms and recent advancements. Adv. Drug Deliv. Rev. doi:10.1016/j. addr.2015.12.012 (2015) (Epub ahead of print). 
9 Mcmahon SJ, Hyland WB, Muir MF et al. Biological consequences of nanoscale energy deposition near irradiated heavy atom nanoparticles. Sci. Rep. 1, 18 (2011).

-• For nanoscale energy deposition.

10 Hainfeld JF, Slatkin DN, Smilowitz HM. The use of gold nanoparticles to enhance radiotherapy in mice. Phys. Med. Biol. 49(18), N309-N315 (2004).

- For first evidence of high- $Z$ element radiosensitization in vivo.

11 Rabin O, Manuel Perez J, Grimm J, Wojtkiewicz G, Weissleder R. An x-ray computed tomography imaging agent based on long-circulating bismuth sulphide nanoparticles. Nat. Mater. 5(2), 118-122 (2006).

12 Mcquaid HN, Muir MF, Taggart LE et al. Imaging and radiation effects of gold nanoparticles in tumour cells. Sci. Rep. 6, 19442 (2016).

13 Lux F, Mignot A, Mowat P et al. Ultrasmall rigid particles as multimodal probes for medical applications. Angew. Chem. Int. Ed. Engl. 50 (51), 12299-12303 (2011).

14 Sancey L, Lux F, Kotb S et al. The use of theranostic gadolinium-based nanoprobes to improve radiotherapy efficacy. Br. J. Radiol. 87(1041), 20140134 (2014).

-• For general overview of the activation and guidance of irradiation by $\mathrm{x}$-ray nanoparticles efficacy in radiosensitization.

15 Bergers G, Benjamin LE. Tumorigenesis and the angiogenic switch. Nat. Rev. Cancer 3(6), 401-410 (2003).

16 Iyer AK, Khaled G, Fang J, Maeda H. Exploiting the enhanced permeability and retention effect for tumor targeting. Drug Discov. Today 11(17-18), 812-818 (2006).

17 Jang SH, Wientjes MG, Lu D, Au JL. Drug delivery and transport to solid tumors. Pharm. Res. 20(9), 1337-1350 (2003).

18 Maeda H, Sawa T, Konno T. Mechanism of tumor-targeted delivery of macromolecular drugs, including the EPR effect in solid tumor and clinical overview of the prototype polymeric drug SMANCS. J. Control. Release 74(1-3), 47-61 (2001).

19 Noguchi Y, Wu J, Duncan R et al. Early phase tumor accumulation of macromolecules: a great difference in clearance rate between tumor and normal tissues. Jpn J. Cancer Res. 89(3), 307-314 (1998).

20 Seymour LW, Miyamoto Y, Maeda $\mathrm{H}$ et al. Influence of molecular weight on passive tumour accumulation of a soluble macromolecular drug carrier. Eur. J. Cancer 31A(5), 766-770 (1995).

21 Kotb S, Detappe A, Lux F et al. Gadolinium-based nanoparticles and radiation therapy for multiple brain melanoma metastases: proof of concept before Phase I trial. Theranostics 6(3), 418-427 (2016).

22 Le Duc G, Roux S, Paruta-Tuarez A et al. Advantages of gadolinium based ultrasmall nanoparticles vs molecular gadolinium chelates for radiotherapy guided by MRI for glioma treatment. Cancer Nanotechnol. 5(1), 4 (2014).

23 Le Duc G, Miladi I, Alric C et al. Toward an imageguided microbeam radiation therapy using gadolinium-based nanoparticles. ACS Nano 5(12), 9566-9574 (2011).

24 Dufort S, Bianchi A, Henry M et al. Nebulized gadoliniumbased nanoparticles: a theranostic approach for lung tumor imaging and radiosensitization. Small 11(2), 215-221 (2015).

25 Detappe A, Kunjachan S, Rottmann J et al. AGuIX nanoparticles as a promising platform for imageguided radiation therapy. Cancer Nanotechnol. 6(1), 4 (2015).

26 Benda P, Someda K, Messer J, Sweet WH. Morphological and immunochemical studies of rat glial tumors and clonal strains propagated in culture. J. Neurosurg. 34(3), 310-323 (1971).

27 Bouchet A, Bidart M, Miladi I et al. Characterization of the 9L gliosarcoma implanted in the Fischer rat: an orthotopic model for a grade IV brain tumor. Tumour Biol. 35(7), 6221-6233 (2014)

- For precise characterization of the 9L rat brain tumor model.

28 Regnard P, Le Duc G, Brauer-Krisch E et al. Irradiation of intracerebral 9L gliosarcoma by a single array of microplanar x-ray beams from a synchrotron: balance between curing and sparing. Phys. Med. Biol. 53(4), 861-878 (2008).

29 Mignot A, Truillet C, Lux F et al. A top-down synthesis route to ultrasmall multifunctional Gd-based silica nanoparticles for theranostic applications. Chemistry (Easton) 19(19), 6122-6136 (2013).

30 Sancey L, Kotb S, Truillet C et al. Long-term in vivo clearance of gadolinium-based AGuIX nanoparticles and their biocompatibility after systemic injection. ACS Nano 9(3), 2477-2488 (2015).

31 Barbier EL, Liu L, Grillon E et al. Focal brain ischemia in rat: acute changes in brain tissue $\mathrm{T} 1$ reflect acute increase in brain tissue water content. NMR Biomed. 18(8), 499-506 (2005).

32 Bianchi A, Dufort S, Lux F et al. Targeting and in vivo imaging of non-small-cell lung cancer using nebulized multimodal contrast agents. Proc. Natl Acad. Sci. USA 111(25), 9247-9252 (2014)

33 Calvo W, Hopewell JW, Reinhold HS, Van Den Berg AP, Yeung TK. Dose-dependent and time-dependent changes in the choroid plexus of the irradiated rat brain. Br. J. Radiol. 60 (719), 1109-1117 (1987).

34 Calvo W, Hopewell JW, Reinhold HS, Yeung TK. Time- and dose-related changes in the white matter of the rat brain after single doses of x rays. Br. J. Radiol. 61(731), 1043-1052 (1988). 